\title{
Modeling IEEE 802.11 DCF with Finite Retry Limits
}

\author{
KVP.Sai Praveen and T.Madhavi \\ Department of ECE, GIT, GITAM University, Visakhapatnam, India
}

\begin{abstract}
The IEEE 802.11 is the dominating standard for wireless local area networks (WLANs). IEEE 802.11 uses Distributed Coordination Function (DCF) protocol with Binary exponential backoff (BEB) algorithm for packet transmission. In WLANs, the performance metrics such as throughput and end-to-end delay greatly depend on packet transmission procedure. The packet transmission procedure can be represented with a Markov chain model. Till now, several Markov chain models have been developed for evaluating the performance of IEEE 802.11 wireless networks. Yet, these models cannot truthfully calculate the performance of the network. Moreover, the existing models suffer with high packet collisions resulting in degradation of throughput particularly under congested environments. In order to overcome this, a new packet transmission procedure for DCF protocol is introduced in this paper considering both packet retry limits and transmission errors. A mathematical analysis is carried out for transmission probability and the throughput of the network is analyzed. Simulation results show significant improvement in throughput of DCF protocol under basic access and request-to-send/clear-to-send (RTS/CTS) access mechanisms using the proposed model when compared with the existing models
\end{abstract}

Keywords: DCF, IEEE 802.11, Markov chain model, Throughput.

\section{Introduction}

Wireless local area networks (WLANs) have been widely used for high-speed wireless applications. A key element to the IEEE 802.11 success is its simple medium access control (MAC) operation based on distributed coordination function (DCF) protocol. DCF designates two methods for packet transmission: the two-way handshaking method called basic access mechanism as well as four-way handshaking method known as request-to-send/clear-to-send (RTS/CTS) mechanism. Readers can see [1] for data transmission procedures using these mechanisms. Before a node/station tries for first packet transmission, it has to sense the medium. If the medium is found to be idle for a minimum time equal to the distributed inter frame space (DIFS), the packet will be transmitted directly. Otherwise, the packet enters into backoff and randomly sets its backoff timer within the range of the contention window $(\mathrm{CW})$. The backoff timer is decremented by one every slot time the medium is sensed to be idle and it is frozen when medium is sensed busy. When it reaches zero, the node starts the next transmission. When the packet is successfully received, the receiver will send an acknowledgment (ACK) after a time equal to the short inter frame space (SIFS). If no ACK is received, the sending node assumes a collision, then doubles its current $\mathrm{CW}$, randomly resets its backoff timer, and retransmits the packet when the timer reaches again 0 . The packet will be discarded if the retry limit reaches its maximum value. This is the main process takes place in packet transmission. This packet transmission procedure can be well represented with a Markov chain model.

Several researchers have studied the performance of IEEE 802.11 DCF by developing the two-state Markov chain models. After the landmark work by Giuseppe Bianchi, who provided an analysis of the saturation throughput of the basic 802.11 protocol assuming a two dimensional Markov model at the MAC layer, many papers have followed the address of this behavior of DCF in a variety of traffic loads and channel transmission conditions [2]. Ziouva et al. introduced a post-backoff stage in the model. To increase the accuracy of the results, busy medium conditions are taken into account in [3]. The IEEE 802.11 performance, primarily the throughput, has been studied in number of papers both analytically and by simulation, but none of them consider finite retry limit and post backoff stage in one [4-7]. Chatzimisios et al. analyze the throughput performance and end-to-end delay by considering packet retry limits [4,5]. Daneshgaran et al. presented a Markov model to analyze the throughput considering transmission errors and capture effects over Rayleigh fading channels [8]. When contention level of the network is high their model is very accurate. In [9], Wu et al. follow the same Markov chain model developed by Bianchi and considers frame retry limits to avoid overestimation of the throughput of 802.11. A post-backoff stage is introduced for maximizing the network throughput in saturation conditions [10]. In [11], Sasi Bhushana Rao et al. developed a new model to predict the accurate performance of the network in erroneous channel conditions. In this model, a backoff delay is introduced between successive packet transmissions to reduce the channel capture effect. Similar to Bianchi's model, the node remains in the last backoff stage until the packet gets transmitted successfully. Using this model, the throughput and delay performance of the network has been greatly improved when compared to the existing models. In this paper, we have modified the Markov chain model presented in [11] considering finite 
retry limits after which the frame is discarded from the transmit queue and a new frame is admitted in the queue. The parameters such as backoff freezing, packet collision errors and channel error conditions are taken into account for this proposed model.

The paper is organized as follows. Alterations made to original DCF are specified in Section II. In Section III, we present the modified Markov chain model and derived the equation for transmission probability. The throughput analysis is carried out in Section IV and finally concluded in Section V.

\section{Alterations Made to Original DCF}

(i) To reduce the contention among nodes, after successful transmission of a packet at any backoff stage, the node waits for a random backoff interval to access the channel again. The advantage of this is to avoid the channel capture. Under saturated conditions, the node selects this interval between $\left(0, W_{0}^{-} 1\right)$ at postbackoff stage where $W_{0}$ is the initial contention window (CW) size.

(ii) In unsaturated conditions, where the packet arrival follows the Poisson's process, the node stays in idle $(-1,0)$ state until the next packet arrives in its queue.

(iii) The packet can be transmitted at any backoff stage when its backoff counter is zero. When the packet arrives at node's buffer and when the channel is idle, it goes from state $(-1,0)$ to state $(0,0)$ and transmits the packet. When the channel is busy, it selects the CW between $\left(0, W_{0}-1\right)$ at $(0,0)$ state.

(iv) The packet will be discarded after reaching maximum retry limits (finite retry limit) defined by the protocol.

\section{Implementation of Modified Markov Chain Model}

Let $b(t)$ and $s(t)$ are the stochastic process representing the backoff timer at time (t) and the backoff stage $(0, \ldots, m)$, respectively. The backoff delay takes the values $\left(0,1, \ldots, W_{i}-1\right)$ where $W_{i}=2^{i} C W_{\min } . W, W_{0}$ and $C W_{\min }$ can be interchangeable. At the first transmission attempt of a packet, $W_{i}$ is set equal to $C W_{\min }$ where $C W_{\min }$ is the minimum contention window size. The contention window will be increased either because of packet collisions or because of transmission errors since a node cannot distinguish a packet collision from a transmission error. If collision occurs in any transmission attempt, the contention window size will get doubled. When this reaches a maximum value $C W_{\max }$ and when the packet transmission fails at this stage $\left(m^{\prime}\right)$ also, the node selects the next contention window size as $C W_{\max }$ until the node reaches the maximum backoff stage, $m$. When the retry of packet transmission reaches the maximum limit as specified by the protocol, the packet will be discarded from queue and a new packet waiting for transmission enters the post back off stage. Therefore, the contention window size is given by (1)

$$
W_{i}= \begin{cases}2^{m} W & i \leq m^{\prime} \\ 2^{m^{\prime}} W & i>m^{\prime}\end{cases}
$$

In order to consider the non-saturated traffic, we define $q$ as the probability of having at least one packet in the node's buffer for its transmission. Similar to Bianchi model [2], the key approximation in the proposed model is that, at each transmission attempt, and irrespective of the number of retransmissions suffered, each packet collides with constant and independent probability $P_{c o l}$ where $P_{c o l}$ is the conditional probability that the transmitted packet encounters a collision. It is assumed that transmission errors because of the imperfect channel can occur with probability $P_{e}$ and the channel is busy with probability $P_{b}$. The collision and transmission error probabilities are assumed to be statistically independent. Here, the state of each node is described by $\{i, k\}$, where $i$ indicates the backoff stage $(0, \ldots, m)$ and $k$ indicates the backoff delay. $P_{e q}$ is the equivalent probability of failed transmission that takes into account the need for a new contention window due to either packet collision $\left(P_{c o l}\right)$ or channel errors $\left(P_{e}\right)$ i.e., $P_{e q}=P_{e}+P_{c o l}-P_{e} P_{c o l}$.

\subsection{Two-state Modified Markov chain model}

The proposed discrete-time Markov chain model considering unsaturated traffic conditions is shown in Fig. 1. In this model, to avoid the channel capture and to reduce the contention among nodes, after successful transmission of a packet at any backoff stage, the node waits for a random backoff interval to access the channel again. Under saturated conditions, the node selects this interval between $\left(0, C W_{\min }\right)$ at $W-1$ stage where $C W_{\min }$ is the minimum contention window size. The selection of the contention window in the next stage depends on the backoff algorithm. The packet can be transmitted at any backoff stage when its backoff counter is zero. The procedure for packet transmission is similar to BEB algorithm except that the delay is introduced between successive packet transmissions. So, a post-backoff stage $(-1, \mathrm{k}), k \in\left\{0 \ldots W_{0}-1\right\}$ with finite retry limits are considered in the proposed Markov model. Section 3.2 describes the backoff state transitions. 
1.2 Backoff State Transitions:

(i) The backoff counter decrements when the node senses the channel idle.

$$
P\{(i, k) \mid(i, k+1)\}=1-P_{b} \quad k \in\left(0, W_{i}-2\right), i \in(0, m)
$$

(ii) The backoff counter freezes when the node senses that the channel is busy.

$$
P\{(i, k) \mid(i, k+1)\}=P_{b} \quad k \in\left(1, W_{i}-1\right), i \in(0, m)
$$

(iii) After each successful transmission, the node with a packet in queue goes to post-backoff stage.

$$
P\{(-1, k) \mid(i, 0)\}=\left(\left(1-P_{b}\right) q\right) / W_{0} \quad k \in\left(0, W_{0}-1\right)
$$

(iv) After unsuccessful transmission at stage $(i-1)$, the node reschedules a backoff delay in the next stage.

$$
P\{(i, k) \mid(\mathrm{i}-1,0)\}=P_{\text {eq }} / W_{i} \quad k \in\left(0, W_{i}-1\right), i \in(1, m)
$$

(v) When the transmission is unsuccessful in all the stages, or after the retry limit is reached, the packet will be discarded/packet is dropped. In this case backoff mechanism is rescheduled for new packet transmission.

$$
P\{(-1, k) \mid(m, 0)\}=1 / W_{0} \quad k \in\left(0, W_{m}-1\right)
$$

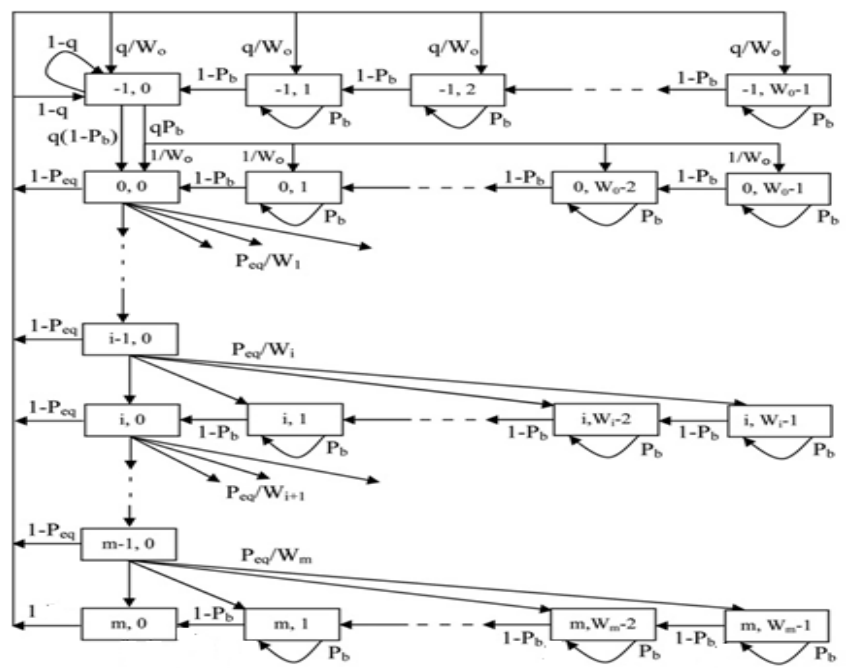

Fig. 1 Proposed Markov chain model

\subsection{Post-Backoff State Transitions:}

(i) After each successful transmission, the node goes to idle state $(-1,0)$ when the queue is empty and waits in that state until the new packet arrives in the queue.

$$
\begin{aligned}
& P\{(-1,0) \mid(i, 0)\}=\left(1-P_{e q}\right)(1-q) \quad i \in(0, m) \\
& P\{(-1,0) \mid(-1,0)\}=1-q
\end{aligned}
$$

(ii) The node with a packet for transmission, goes to $(0,0)$ state when the channel is free and then transmits the packet.

$$
P\{(0,0) \mid(-1,0)\}=q\left(1-P_{b}\right)
$$

(iii) The node with a packet for transmission, selects a backoff stage when the channel is busy.

$$
P\{(0, k) \mid(-1,0)\}=\frac{q P_{b}}{W_{0}} \quad k \in\left(0, W_{0}-1\right)
$$

(iv) In the post-backoff stage, the backoff counter decrements when the node senses the channel idle and freezes when the channel is busy.

$$
\begin{array}{ll}
P\left\{(-1, k) \mid(-1, k+1)=\left(1-P_{b}\right)\right. & k \in\left(0, W_{0}-2\right) \\
P\{(-1, k) \mid(-1, k)\}=P_{b} & k \in\left(-1, W_{0}-1\right)
\end{array}
$$

The probability that a node occupies a given state $\{i, k\}$ at any discrete time slot is $b_{i, k}=\operatorname{limt} \rightarrow \infty P\{s(t)=i, b(t)$ $=k\}$ where $i, k$ are integers and $-1 \leq i \leq m, 0 \leq k \leq W_{i}-1$. In steady state, the following relations are valid:

$$
b_{i, k}=\frac{W_{i}-k}{W_{i}} \frac{P_{e q}^{i}}{1-P_{b}} b_{0,0} \quad k \in\left(1, W_{i}-1\right) ; i \in(1, m-1)
$$




$$
\begin{aligned}
& b_{m, o}=\frac{P_{e q}^{m}}{1-P_{e q}} b_{0,0} \\
& b_{m, k}=\frac{W_{i}-k}{W_{i}} \frac{P_{e q}^{m}}{\left(1-P_{b}\right)} b_{0,0}
\end{aligned}
$$

In the above Markov chain model, after successful transmission at any backoff stage, the node directly enters state $(-1,0)$ when there are no packets to be transmitted and keeps iterating in that state until the arrival of new packet. The stationary probability to be in state $b_{-1,0}$ can be evaluated as

$$
b_{-1,0}=\frac{1-q}{q} b_{0,0}
$$

After successful transmission at any backoff stage, when the node is ready to transmit the next packet, the node enters state $(-1, k)$ to provide some backoff delay between these packets to avoid channel capture. So the stationary probability to be in state $b_{-1, k}$ obtained is

$$
b_{-1, k}=q\left(\frac{W_{0}-k}{W_{0}}\right) \frac{1}{1-P_{b}} b_{0,0} \quad k \in\left(1, W_{0}-1\right)
$$

And the stationary probability that the node to be in state $b_{0, \mathrm{k}}$ is

$$
b_{0, k}=\frac{P_{b}(1-q)}{1-P_{b}} \frac{W_{0}-k}{W_{0}} b_{0,0}
$$

According to probability conservation relation, total probability is equal to one. Therefore

$$
\sum_{i=-1}^{m} b_{i, o}+\sum_{i=-1}^{m} \sum_{k=1}^{W_{i}-1} b_{i, k}=1
$$

\subsection{Probability of Transmission, $\tau$}

Let $\tau$ be the probability with which a node transmits a packet in a randomly chosen slot time. The node transmits the packet when the backoff counter reaches the value of zero. Then the equation for $\tau$ becomes

$$
\tau=\sum_{i=0}^{m} b_{i, 0}=\frac{1}{1-P_{e q}} b_{0,0}
$$

On solving the above equations, $\tau$ can be expressed as

$$
\tau=\left\{\begin{array}{c}
\frac{2 q\left(1-P_{b}\right)\left(1-2 P_{e q}\right)}{\left[2(1-q)\left(1-P_{b}\right)\left(1-P_{e q}\right)\left(1-2 P_{e q}\right)\right]+\left[2 q\left(1-P_{b}\right)\left(1-2 P_{e q}\right)\right]+\left[q\left(1-P_{e q}\right)\left(1-2 P_{e q}\right)(W-1)\left(P_{b}(1-q)+q\right)\right]} \cdots m<m^{\prime} \\
{\left[\begin{array}{c}
\left.P_{e q}^{m} q\left(2^{m} W-1\right)\left(1-2 P_{e q}\right)\right]+\left[2 q W P_{e q}\left(1-P_{e q}\right)\left(1-\left(2 P_{e q}\right)^{m-1}\right)\right]-\left[q P_{e q}\left(1-2 P_{e q}\right)\left(1-P_{e q}^{m-1}\right)\right] \\
\frac{2 q\left(1-P_{b}\right)\left(1-2 P_{e q}\right)}{\left[2(1-q)\left(1-P_{b}\right)\left(1-P_{e q}\right)\left(1-2 P_{e q}\right)\right]+\left[2 q\left(1-P_{b}\right)\left(1-2 P_{e q}\right)\right]+\left[q\left(1-P_{e q}\right)\left(1-2 P_{e q}\right)(W-1)\left(P_{b}(1-q)+q\right)\right]} \cdots m>m^{\prime} \\
{\left[2 q W P_{e q}\left(1-P_{e q}\right)\left(1-\left(2 P_{e q}\right)^{m^{\prime}}\right)\right]-\left[q P_{e q}\left(1-2 P_{e q}\right)\left(1-P_{e q}^{m^{\prime}}\right)\right]+\left[q W\left(1-P_{e q}\right)\left(2 P_{e q}\right)^{m^{\prime}+1}\left(1-\left(2 P_{e q}\right)^{m^{\prime}-m}\right)\right]} \\
-\left[q\left(1-2 P_{e q}\right)\left(P_{e q}\right)^{m^{\prime \prime}+1}\left(1-\left(P_{e q}\right)^{m-m^{\prime}}\right)\right]
\end{array}\right.}
\end{array}\right.
$$

In the above equation, the transmission probability $\tau$ depends on $P_{c o l}$ and $P_{b}$. In (19), under saturated traffic conditions $(q \rightarrow 1)$ and when $m=0$, that is when no exponential backoff is considered and assuming the packet transmission errors are only because of collisions and $P_{b}=0, \tau$ reduces to

$$
\tau=\frac{2}{W+1}
$$

This is similar to the equation for the constant backoff window problem which shows that the transmission probability is independent of the collision probability. Busy $P_{b}$ and collision probabilities $P_{c o l}$ are important parameters in evaluating the performance of IEEE 802.11 system and are given as

$$
\begin{aligned}
& P_{t r}=P_{b}=1-(1-\tau)^{n} \\
& P_{c o l}=1-(1-\tau)^{n-1}
\end{aligned}
$$

where $n$ is number of contending nodes. Equations (19), (21) represent a non-linear system with the three unknowns $\tau, P_{c o l}$ and $P_{b}$, which can be solved by numerical methods and has a unique solution. Note that $P_{c o l} \in$ $[0,1], P_{b} \in[0,1]$ and $\tau \in[0,1]$. 


\subsection{Frame error probability, $P_{e}$}

The performance analysis of the proposed model is done using the network parameters of IEEE $802.11 \mathrm{~b}$ protocol since it is easier to compare with the existing models, even though the model suits for any IEEE 802.11 family. The IEEE $802.11 \mathrm{~b}$ supports data rates of 1, 2, 5.5 and $11 \mathrm{Mbps}$. In this, the physical layer convergence procedure (PLCP) preamble and header are transmitted using differential binary phase shift keying (DBPSK) modulation at a transmission rate of $1 \mathrm{Mbps}$. The MAC protocol data unit (MPDU) is transmitted with the rate depending on the modulation used. Due to imperfect channels, the transmitted frame will get corrupted and the frame error probability is affected by the bit error probability and the size of the frame.

The frame error probability, $P_{e}$ is defined as (11)

$$
P_{e}=1-\left(1-P_{P H Y_{-} \text {eror }}\right)\left(1-P_{\text {MAC_eror }}\right)
$$

where $P_{\text {PHY_error }}$ is the physical layer (PHY) overhead error probability and $P_{\text {MAC_error }}$ is the MPDU error probability.

The PHY and MAC layer overhead probabilities depend on the bit error probabilities as given below

$$
P_{P H Y_{-} \text {error }}=1-\left(1-P_{b 1}\right)^{24 \times 8}
$$

Where $P_{b l}$ is the 'bit error probability' or probability of error of the PLCP preamble and header (24 bytes) occurs during the transmission of physical layer overhead

$$
P_{M A C_{-} \text {error }}=1-\left(1-P_{b 2}\right)^{(28+M S D U) 8}
$$

Where $P_{b 2}$ is the 'bit error probability' or probability of error of the MPDU occurs during the transmission of MAC header (28 bytes) and MAC service data unit (MSDU). The equations of $P_{b 1}$ and $P_{b 2}$ can be found in [12].

\section{Throughput Analysis}

The performance of the wireless communication network can be evaluated in terms of system throughput, probability of collision and so on. The average throughput is the ratio between the total data received and the total delay incurred. The core contribution of this paper is analytical evaluation of the system throughput, by considering non-saturated traffic conditions and effect of channel errors during packet transmission under Rayleigh fading channel environment. A packet is transmitted successfully when the packet encounters no collisions and no channel errors are introduced during transmission. In this paper, the throughput analysis is carried out by considering transmission errors under finite load conditions and compared with the existing models under basic and RTS/CTS access mechanisms. When the data passes through the communication channel, it is corrupted by the noise. Unsuccessful transmission occurs when more than one user simultaneously transmit the packets that collides with each other or the data packets may be corrupted at the receiver because of erroneous channels. In both the cases, the acknowledgment (ACK) will not be received by the transmitting node and it reschedules the backoff procedure.

Let the normalized system throughput $S$, defined as the fraction of time the channel is used to transmit the payload bits successfully. The expected time per slot $E[S t]$ can be calculated by taking the successful transmission slot time with the probability $P s\left(1-P_{e}\right)$, unsuccessful transmission slot time because of collision with the probability $\left(1-P_{s}\right)$, unsuccessful transmission slot time because of channel errors with probability $P s T e$ and idle slot time with probability $(1-P t r)$.

Now, the equation for $E\left[S_{t}\right]$ can be written as

$$
E\left[S_{t}\right]=P_{t r} P_{s}\left(1-P_{e}\right) T_{\text {success }}+P_{t r}\left(1-P_{s}\right) T_{\text {collision }}+\left(1-P_{t r}\right) \text { slot_time }+P_{t r} P_{s} P_{e} T_{e}
$$

In the above equation $P_{s}$ is the probability that a transmission occurring on the channel is successful is given by the probability that exactly one node transmits on the channel, conditioned on the fact that at least one node transmits and is given as

$$
P_{s}=\frac{n \tau(1-\tau)^{n-1}}{P_{t r}}=\frac{n \tau(1-\tau)^{n-1}}{1-(1-\tau)^{n}}
$$

Following a similar thought from [11], we can express the normalized saturation throughput of IEEE 802.11 DCF when the channel errors are considered as follows:

$$
S=\frac{P_{t r} P_{s}\left(1-P_{e}\right) E[P]}{P_{t r} P_{s}\left(1-P_{e}\right) T_{\text {sucess }}+P_{t r}\left(1-P_{s}\right) T_{\text {collision }}+\left(1-P_{t r}\right) \text { SlotTime }+P_{t r} P_{s} P_{e} T_{e}}
$$


In the above equation, slot time is the idle slot time. $T_{\text {success }}, T_{\text {collision }}$ and $T_{e}$ are the average times that the channel is sensed busy because of a successful transmission, collision and transmission errors. Here, $T_{\text {collision }}$ and $T_{e}$ are assumed to be same. $E[P]$ is the average packet payload size.

The equations for $T_{\text {success }}$ and $T_{\text {collision }}$ using basic and RTS/CTS access mechanisms are given below

$$
\begin{aligned}
& T_{\text {success(basic) }}=D I F S+T_{P A C K E T}+\delta+S I F S+T_{A C K}+\delta \\
& T_{\text {collision(basic) }}=T_{e}=T_{\text {PACKET }}+\delta+A C K_{-} \text {Timeout } \\
& T_{\text {success }(R T S / C T S)}=D I F S+T_{R T S}+\delta+S I F S+T_{C T S}+\delta+S I F S+T_{P A C K E T}+\delta+S I F S+T_{A C K}+\delta(31) \\
& T_{\text {collision(RTS/CTS })}=T_{e}=D I F S+T_{R T S}+\delta+S I F S+T_{C T S}+\delta+S I F S
\end{aligned}
$$

Where DIFS is the distributed interframe space period, SIFS is the short interframe space period, $T_{\text {PACKET }}$ is the time taken to transmit the data including PHY and MAC headers. $T_{R T S}, T_{C T S}$ and $T_{A C K}$ are the timings required to transmit RTS, CTS and ACK frames, respectively, and $\delta$ is the propagation delay. The ACK_Timeout $=$ SIFS + $T_{A C K}+D I F S$.

In the literature, many researchers have analysed the performance of the wireless networks under saturated traffic conditions. On the other hand, the network does not yield best performance at saturated condition and wide-ranging of research has been undertaken to avoid the network from saturation [13]. So by considering effects of $q$ and the analysis is done to a random input rate, which creates a more challenging problem. To analyse the performance of unsaturated wireless networks, a parameter $\lambda$ is used which represents the rate at which packets arrive at the node's buffer from the upper layers and measured in packets per second (Pkts/s). If the traffic arrives in a Poisson distribution with small buffer size, the probability $q$ can be well approximated as [8]

$$
q=1-e^{-\lambda E\left[S_{t}\right]}
$$

A more accurate model can be derived upon considering different values of $q$ for each backoff state. However, a reasonable solution consists in using a mean probability valid for the whole Markov model derived from $E[S t]$. Now, $E[S t]$ can be used to calculate the probability $q$. The probability for $k$ packet arrivals in a generic time $\mathrm{T}$ is given by

$$
P\{a(T)=k\}=e^{-\lambda T} \frac{(\lambda T)^{k}}{k !}
$$

From the above equation, the relation of $E[S t]$ and $q$ can be written as

$$
q=1-P\left\{a\left(E\left[S_{t}\right]=0\right\}=1-e^{-\lambda E\left[S_{t}\right]}\right.
$$

The throughput as a function of packet rate using basic and RTS/CTS access mechanisms is observed. Fig. 2 evaluates the throughput as a function of $n$ under basic access mechanism when $P_{e}=10^{-1}$. When the number of nodes is 50, the proposed model gives the throughput of $1.7 \%$ and $4 \%$ higher for $m=4$ and $m=7$ respectively under basic access mechanism when compared with the reference model [11].
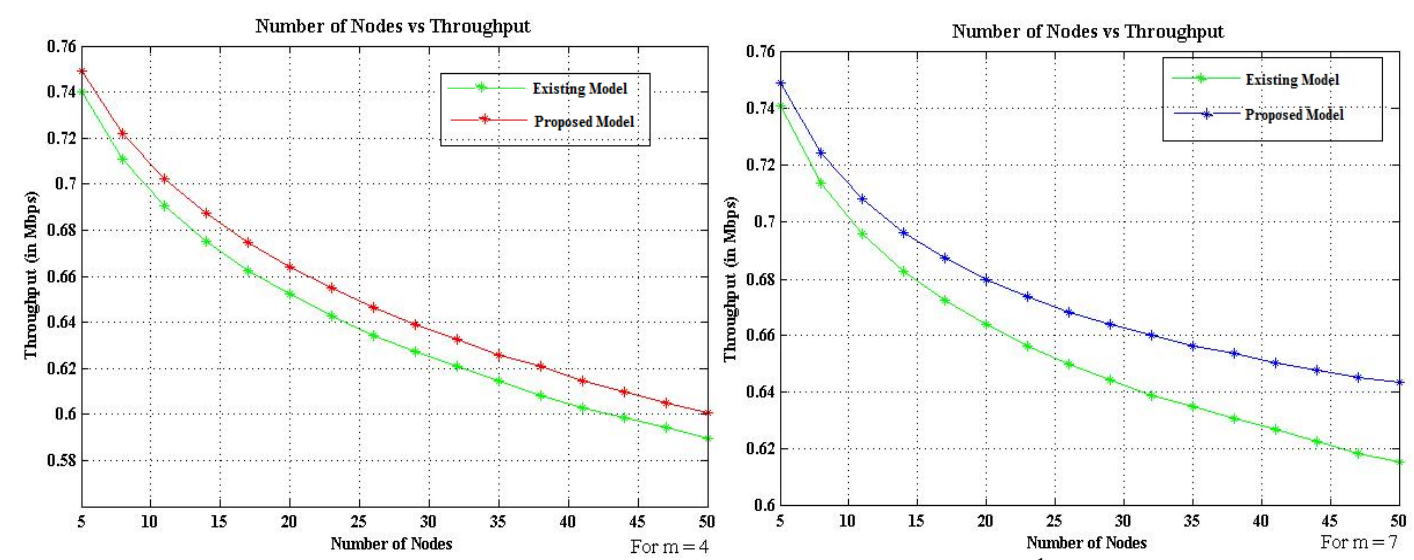

Fig. 2 Throughput as a function of nodes when $\lambda=50 \mathrm{Pkts} / \mathrm{s}$ and $P_{e}=10^{-1}$ under Basic Access Mechanism 

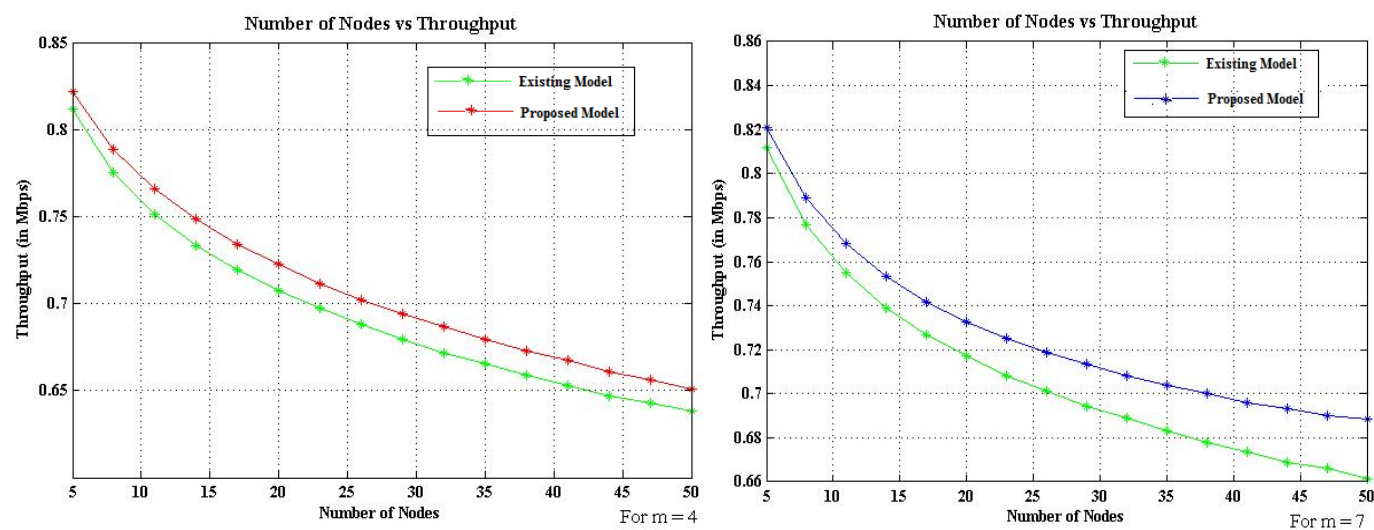

Fig. 3 Throughput as a function of nodes when $\lambda=50 \mathrm{Pkts} / \mathrm{s}$ and $P_{e}=10^{-3}$ under Basic Access Mechanism

The throughput as a function of $n$ under basic access mechanism is shown in Fig. 3 when $P_{e}=10^{-3}$. The proposed model gives the throughput of $1.6 \%$ and $4.5 \%$ higher when compared with the reference model [11] for $m=4$ and $m=7$ respectively under basic access mechanism when the number of nodes is 50 .
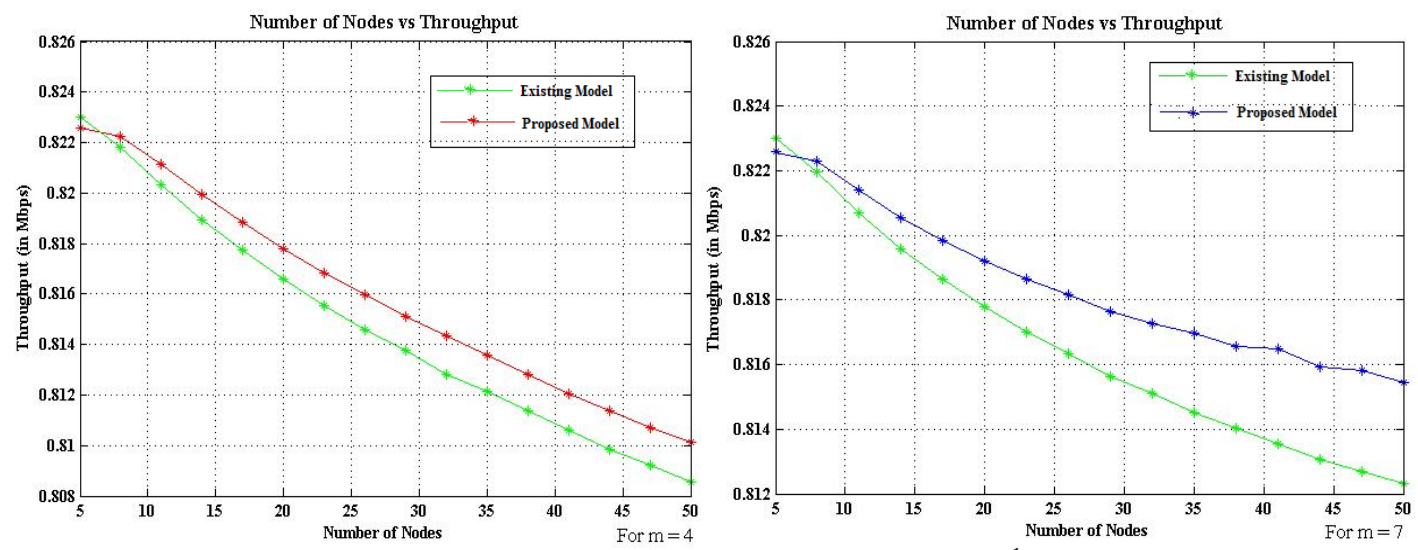

Fig. 4 Throughput as a function of nodes when $\lambda=50 \mathrm{Pkts} / \mathrm{s}$ and $P_{e}=10^{-1}$ under RTS/CTS Mechanism

Fig.4 evaluates the throughput as a function of $n$ under RTS/CTS access mechanism when $P_{e}=10^{-1}$. When the number of contending nodes is less than 7, the throughput obtained using the proposed model is somewhat less compared with the reference model but the performance of the proposed model is better when compared with the existing model.

The throughput as a function of $n$ under RTS/CTS access mechanism is plotted in Fig. 5 when $P_{e}=10^{-3}$. It is observed that even under RTS/CTS mechanism the performance of the proposed model is better when compared with the reference model [11]. Moreover, performance is highly dependent on the network size, increasing network size results in a decrease in throughput. Notations used for analysis is listed in Table 1 and the simulation parameters used for performance evaluation is listed in Table 2.
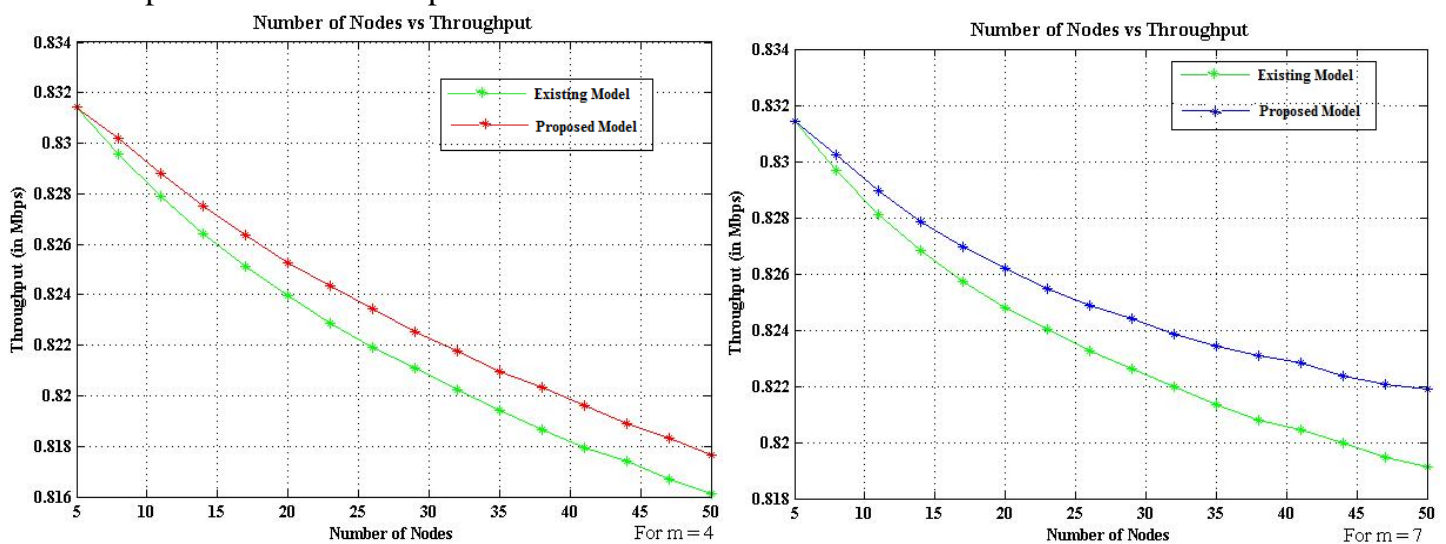

Fig. 5 Throughput as a function of nodes when $\lambda=50 \mathrm{Pkts} / \mathrm{s}$ and $P_{e}=10^{-3}$ under RTS/CTS Mechanism 
Table $1 \quad$ Notations used in analytical analysis

\begin{tabular}{ll}
\hline Notation & \multicolumn{1}{c}{ Description } \\
\hline$P_{b}, P_{t r}$ & Probability that the node at the backoff stage senses the channel busy \\
$P_{s}$ & Probability of successful transmission \\
$P_{c o l}$ & Probability of transmission collision \\
$P_{e}$ & Probability of transmission failure due to imperfect channel conditions \\
$P_{e q}$ & Equivalent probability of failed transmission \\
$W_{0}, W$ & Initial contention window size \\
$T$ & The probability that a station transmits a packet in a randomly chosen slot time \\
$m$ & Maximum backoff stage \\
$m^{\prime}$ & Contention Window increasing factor \\
$n$ & Number of contending nodes \\
$q$ & The probability of having at least one packet in the stations buffer to transmit \\
$\lambda$ & Packet arrival rate \\
$E[P]$ & Average packet payload size \\
$E\left[S_{t}\right]$ & Expected time per slot \\
$D I F S$ & Distributed interframe space period \\
$S I F S$ & Short interframe space period \\
$\delta$ & Propagation delay \\
$T_{A C K}, T_{R T S} \& T_{C T S}$ & Transmission time of ACK, RTS and CTS frames \\
$T_{P A C K E T}$ & Transmission time of ACK, RTS and CTS frames \\
$T_{\text {success }}$ & Average time that the channel is sensed busy because of successful transmission \\
$T_{\text {collision }}$ & Average time that the channel is sensed busy due to collisions \\
$T_{e}$ & Average time that the channel is sensed busy due to transmission error \\
\hline &
\end{tabular}

Table 2 Simulation Parameters

\begin{tabular}{|ll|lr|}
\hline Channel Bit Rate & 1 Mbps & DIFS & $50 \mu \mathrm{s}$ \\
PHY header & 24 bytes & SIFS & $10 \mu \mathrm{s}$ \\
MAC header & 28 bytes & Slot Time & $20 \mu \mathrm{s}$ \\
RTS & 20 bytes + PHY header & Propagation Delay, $\delta$ & $1 \mu \mathrm{s}$ \\
CTS & 14 bytes + PHY header & $\mathrm{CW}_{\min }\left(\mathrm{w}_{\min }\right)$ & $31(\mathrm{slots})$ \\
ACK & 14 bytes + PHY header & $\mathrm{CW}_{\max }\left(\mathrm{w}_{\max }\right)$ & 1023 (slots) \\
\hline
\end{tabular}

\section{Conclusions}

In this paper, a new packet transmission procedure for IEEE 802.11 wireless networks is developed for evaluation of throughput considering finite retry limits. An analytical model is developed to calculate the transmission probability. The throughput under non-saturated and erroneous channel conditions is analyzed. The throughput of the proposed model is found to be significantly improved when compared with the existing model. Finally, the procedure for data transmission explained in this paper enhances the throughput performance compared with existing DCF protocol used in WLAN.

\section{References}

[1]. IEEE Standard for Wireless LAN Medium Access Control (MAC) and Physical Layer (PHY) Specifications, IEEE 802.11b, 1999.

[2]. G. Bianchi, Performance analysis of the IEEE 802.11 distributed coordination function, IEEE J. on Selected Areas in Comm., Vol. 18(3), pp. 535-547, March 2000.

[3]. Ziouva.E and Antonakopoulos.T, CSMA/CA performance under high traffic conditions throughput and delay analysis, Comput. Commun, 25(3), pp. 313-321, 2002.

[4]. P. Chatzimisios, A.Boucouvalas and Vitsas.V, Throughput and delay analysis of IEEE 802.11 protocol, IWNA, pp. 168-174,2002

[5]. P. Chatzimisios, A.Boucouvalas and Vitsas. V, IEEE 802.11 Packet Delay: A Finite Retry Limit Analysis, Proc. IEEE Globecom, Vol. 2, pp. 950-954, 2003.

[6]. Z.Hadzi Velkov and B.Spasenovski, Saturation Throughput - Delay Analysis of IEEE 802.11 DCF in Fading Channel, IEEE Transactions on Wireless Comm, vol 11(3), pp7803-7802, June2003.

[7]. Z.Hadzi Velkov and B. Spasenovski, On the Capacity of IEEE 802.11DCF with Capture in Multipath-faded Channels, Int. Journal of Wireless Information Networks, vol. 9(3), Kluwer Academic, July 2002.

[8]. F.Daneshgaran, M. Laddomada, F. Mesiti and M. Mondin, Unsaturated Throughput Analysis of IEEE 802.11 in Presence of Non Ideal Transmission Channel and Capture Effects, IEEE Transactions on Wireless Comm., Vol. 7(4), April 2008.

[9]. H.Wu, Y.Peng, K.Long and Cheng.S, Performance of reliable transport protocol over IEEE 802.11 wireless LAN: Analysis and enhancement. Proc. IEEE INFOCOM, vol. 2, pp. 599-607, 2002. 
[10]. Anouar. H, and Bonnet.C, Optimal constant-window backoff scheme for IEEE 802.11 DCF in single-hop wireless networks under finite load conditions", Wirel. Pers. Commun., 43, pp. 1583-1602, 2007.

[11]. Sasi Bhushana Rao Gottapu, Madhavi Tatineni and MNVSS Kumar, Performance analysis of collision alleviating distributed coordination function protocol in congested wireless networks- a Markov chain analysis, IET Networks, Vol. $2(4)$. Dec 2013.

[12]. Mahasukhon.P, Hempel.M, Sharif.H, Zhou.T, and Chen.H, BER analysis of $802.11 \mathrm{~b}$ networks under mobility, IEEE Int. Conf. on Communications, ICC '07, pp. 4722-4727, June 2007.

[13]. Zhao.H, Wei.J, Wang.S and Xi.Y, Available bandwidth estimation and prediction in ad hoc networks, Mobile Ad-Hoc Networks: Protocol Design, National University of Defense Technology, China, pp. 61-84, January 2011. 